\title{
Temporal expectations affect retrospective temporal judgments: evidence in favour of assimilation effects
}

\author{
Florian Klapproth \\ University of Luxembourg, Luxembourg, Luxembourg
}

Email address:

florian.klapproth@uni.lu (F. Klapproth)

\section{To cite this article:}

Florian Klapproth. Temporal Expectations Affect Retrospective Temporal Judgments: Evidence in Favour of Assimilation Effects. Psychology and Behavioral Sciences. Vol. 2, No. 2, 2013, pp. 59-65. doi: 10.11648/j.pbs.20130202.16

\begin{abstract}
A study containing four experiments provided evidence in favour of assimilation effects in retrospective duration judgments due to temporal expectations. In this study, the participants did not know in advance that they would have to reproduce the duration of a target interval. Temporal expectations were induced prior to the target interval by the repeated presentation of a visually filled interval (the expectancy interval). Both the duration of the expectancy interval and the number of presentations of that interval were varied between subjects. The experiments showed a clear assimilation effect of temporal expectations on reproduced duration, indicated by judged durations strongly resembling the duration of the expectancy interval. This effect increased with the magnitude of the difference between the expectancy interval and the target interval, and with the number of repetitions of the expectancy interval. Results were discussed with reference to Helson's adaptation-level theory.
\end{abstract}

Keywords: Assimilation Effect; Temporal Expectations; Temporal Reproduction; Retrospective Duration Judgment; Experiment

\section{Introduction}

Though expectations are ubiquitous and do certainly guide our behaviour, there is still only a small number of studies that investigated the effects of expectations on temporal judgments. Temporal expectations may be established either incidentally via a temporally regular structure, or deliberately by informative (verbal) cues [1]. Some of the oldest experiments addressing the effect of expectancy on time estimation were concerned with determinants of the temporal indifference interval (e. g., $[2,3,4,5]$ ). In these experiments, temporal judgments often resembled the duration of preceding intervals, which appeared to establish a frame of reference for succeeding temporal judgments.

The role expectations might play in temporal judgments has been re-examined in recent years. For example, within a prospective timing task, [6] presented participants sequences of tones, with inter-onset intervals (IOIs) separating the tones being equal. The two last tones of the sequence confined the standard interval, which was longer than, shorter than, or equal to the IOIs. The standard interval was succeeded by a comparison interval, of which the duration had to be judged. Results showed a significant effect of the preceding IOIs on duration judgment: If the IOIs were longer than the standard, the standard was judged on average as being longer than the comparison. If, however, the IOIs were shorter than the standard, the standard was likely to be judged as being shorter than the comparison. In other words, the preceding IOIs appeared to alter the subjective duration of the standard, with long IOIs resulting in a prolonged internal standard, and with short IOIs resulting in a shortened internal standard. Similarly, [7] examined how an added sequence of temporal intervals that preceded the to-be-judged intervals affected a prospective same-different task. Results indicated that temporal judgments were most accurate when all intervals preceding the to-be-judged interval were equal in duration to the to-be-judged interval.

Whereas there is some evidence that temporal expectations lead to assimilation effects in prospective temporal judgments, fewer studies were concerned with examining how temporal expectations do affect time estimation in retrospective. In contrast to prospective judgment tasks, the participants in retrospective judgment tasks do not know in advance that they will be required to estimate time. Most of the results from retrospective timing tasks were gained from studies that investigated the experience of waiting times. For example, [8] let her participants wait for the beginning of an experiment slightly more than four minutes. In one experi- 
mental condition the participants were provided with the (false) information that they had to wait for two minutes. In another condition, no information was given as to the expected duration of the wait. Perceived duration was shorter in the former condition than in the latter one. Similar results were obtained in a study conducted by [9] whose participants waited for being registered for a computer course. One group received (correct) information about the expected length of the wait, whereas the other group did not get any information about the wait's duration. The participants who received waiting-time information judged the waiting time more precisely than participants without information. Recently, [10] conducted a series of experiments which examined whether temporal expectancies affected retrospective judgments of durations. One of the main results of this study was that the duration of the waiting period positively correlated with the reproductions of the target duration, that is, the longer (or shorter) the waiting period was, the longer (or shorter) were the reproductions of the target duration.

A theory that might be capable of explaining assimilation effects in duration judgments due to temporal expectations is the adaptation-level theory $[11,12,13]$. According to this theory, all relevant stimuli that are presented within an experimental session establish a frame of reference that is used for later judgments $[14,15,16]$. This frame of reference is the adaptation level, and several models for its quantification had been proposed. For example, Helson [11] suggested to conceive the adaptation level as the geometric mean of all stimuli presented, whereas others (and even Helson some years later) proposed the arithmetic mean as being a better estimate for the adaptation level $[12,17,18]$.

Adaptation-level theory has been applied to explaining temporal judgments, predominantly within prospective paradigms (e. g., [19, 20, 21]). However, the main principle of adaptation-level theory, namely that judgments of stimuli (durations) are affected by a frame of reference, is valid for both judgment paradigms. According to most theories of human timing (e. g., [22]), temporal judgments within either paradigm involve at least three stages of information processing: (1) the encoding of the to-be-judged duration, (2) the storage of a reference duration that is going to serve as a standard for the judgment, and (3) a comparison between the standard and the to-be-judged duration. Whereas the first stage should to be rather unaffected by stimuli that precede the to-be-judged stimulus, storage of a reference duration and the subsequent comparison process are not. The reference duration may be composed of durations that preceded the to-be-judged duration, thus forming some kind of average duration which will serve as a standard in later comparisons. Accordingly, judgment of duration will strongly depend on this standard established by preceding durations.

The expected duration of a to-be-judged stimulus may also be regarded as a frame of reference for temporal judgments. Contents of reference memory might be changed according to temporal expectations by replacing (or at least modifying) the internal standard by the average of encountered durations $[23,24,25]$. In a similar vein, some authors
[26] assume that the mean of preceding intervals determines an expected value for the internal standard.

With the present study, adaptation-level theory was used for explaining effects of temporal expectancy on retrospective temporal judgments. Temporal expectancy was established by the succession of intervals of constant duration prior to the to-be-judged interval. Although some studies have shown that assimilation effects in temporal judgments due to temporal expectancy do occur in retrospective judgments (e. g., $[8,9,10])$, some problems are still unresolved. For instance, it is unclear whether the strength of expectancy or the difference between expected duration and target duration affects temporal judgments. Since the adaptation-level is conceived of as the average of experienced durations, more repetitions of preceding intervals should result in an adaptation level being more similar to the preceding intervals. Hence, the assimilation effect should be more pronounced, the more intervals preceded the target duration. With respect to the difference between the duration of preceding intervals and the duration of the target interval, it was supposed that according to adaptation-level theory larger differences should elicit larger assimilation effects.

The first two experiments were conducted primarily in order to replicate the results of [10], with only marginal changes of the experimental procedure. In addition, the effect of varying numbers of expectancy intervals and of varying differences between the duration of expectancy and target intervals on the assimilation effect was examined with Experiment 3 and 4.

\section{Experiment 1}

\subsection{Method}

\subsubsection{Participants}

In this experiment nine men and 21 women participated, with a mean age of $40.2(S D=12.7)$ years. The participants were randomly and in equal number $(n=15)$ assigned to the two conditions of the experiment.

\subsubsection{Materials and Procedure}

The stimuli were presented and responses were recorded by a laptop running with a $1-\mathrm{GHz}$ processor. The experimental program was written with E-Prime ${ }^{\circledR}$, Version 1.1. The participants were given an attention test [27] which served as an activity period within the experiment. In this test, the participants had to mark the letter " $\mathrm{d}$ ", combined with two dashes, as frequently as possible within an array of similar letters. The participants began with the activity after having heard a high-pitched $(800 \mathrm{~Hz})$ tone and stopped with this activity after the presentation of a low-pitched $(400 \mathrm{~Hz})$ tone, presented via headphones. Each tone lasted for $500 \mathrm{~ms}$. After the period of activity a waiting period (the expectancy interval) followed in which the participants watched a grey square $(9 \times 9 \mathrm{~cm})$ presented in the middle of a computer monitor screen. Then, another period of activity followed, which was followed by another expectancy interval, and so forth. This sequence of activity and waiting was repeated 
nine times. The last waiting period within the sequence was the target duration which was to be judged by the participants. Thereafter, the participants judged the target duration by using a reproduction task. After the onset of a visual stimulus they pressed a button on the computer keyboard to terminate the stimulus when experiencing matching duration of that stimulus and the target interval.

Both the duration of the target interval and the duration of the period of activity were kept constant in all conditions (15 s). The duration of the expectancy intervals presented prior to the target interval was either $10 \mathrm{~s}$ or $20 \mathrm{~s}$.

\subsection{Results and Discussion}

Figure 1 shows the results of Experiment 1.

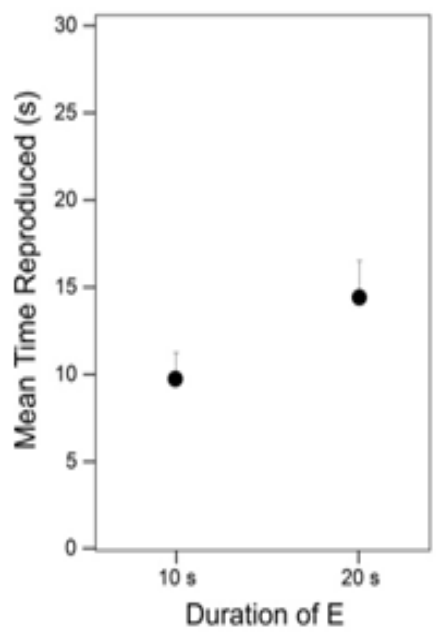

Figure 1. Mean reproduction times obtained from Experiment 1, plotted against the different $E$ durations (on the $x$-axis). Error bars mean standard errors.

As indicated by Figure 1, reproductions were largely affected by the expectancy interval $E$. With $E=10$ s, reproductions were rather short $(M=9.49 \mathrm{~s}, S D=4.30)$, whereas with $E=20 \mathrm{~s}$, reproductions were much longer on average $(M=13.97 \mathrm{~s}, S D=6.39)$. The difference between both mean reproductions was significant, $t(28)=-2.25, p=.032, \eta^{2}$ $=.153$.

The results obtained from Experiment 1 show a clear assimilation effect of reproduced durations towards the expected duration. If the expected duration was longer than the target duration, reproductions were on average longer than if the expected duration was shorter than the target duration.

\section{Experiment 2}

The first aim of Experiment 2 was to reproduce the assimilation effect obtained from the previous experiment. Moreover, with Experiment 2, a zero-assimilation condition was established wherein the expectancy interval was equal to the target interval. It was assumed that no assimilation effect should occur when the target interval equals the expectancy interval.

\subsection{Method}

\subsubsection{Participants}

In this experiment 16 men and 23 women participated, having a mean age of 29.8 years $(S D=11.4)$. The participants were randomly and in equal number assigned to the three conditions of the experiment.

As in Experiment 1, the duration of the target interval and the duration of the period of activity were kept constant in all conditions $(15 \mathrm{sec})$. The duration of the expectancy interval was $10 \mathrm{sec}$ or $20 \mathrm{sec}$. Additionally, a third condition was established which entailed an expectancy interval that equalled the target interval $(15 \mathrm{sec})$.

\subsubsection{Materials and Procedure}

All stimuli used (except for the third condition), as well as the procedure, were identical to those used in the previous experiment.

\subsection{Results and Discussion}

As can be seen in Figure 2, reproduced durations varied according to the expectancy interval. Reproductions were shortest with $E=10 \mathrm{~s}(M=7.64 \mathrm{~s}, S D=2.91)$, longest with $E$ $=20 \mathrm{~s}(M=15.29 \mathrm{~s}, S D=5.4)$, and lay in between with $E=$ $15 \mathrm{~s}(M=10.06 \mathrm{~s}, S D=2.38)$. Analysis of variance with duration of expectancy interval as between-subjects factor produced a significant main effect, $F(2,36)=13.75, p<.001$ $\eta^{2}=.433$. Bonferroni post hoc tests revealed significant differences of reproductions between the $E=10$ s condition and the $E=20 \mathrm{~s}$ condition, and between the $E=15 \mathrm{~s}$ condition and the $E=20$ s condition.

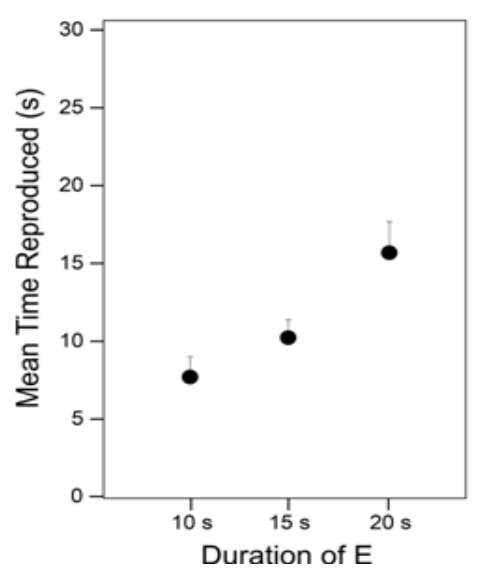

Figure 2. Mean reproduction times obtained from Experiment 2, plotted against the different $E$ durations (on the $x$-axis). Error bars mean standard errors.

As assumed, assimilation effects in temporal reproductions were observed in Experiment 2 in conditions wherein the target interval was different from the expectancy interval. Temporal reproductions were shorter with short expectancy intervals and longer with long expectancy intervals. In case of the expectancy interval being equal to the target interval, mean reproductions were between the reproductions of the remaining conditions. However, although no assimilation 
was expected to occur in this condition, mean reproductions were shorter than the target interval. This result appears to reflect a general trend to underestimate presented target intervals.

\section{Experiment 3}

In Experiment 3, the expectancy intervals preceding the target duration differed only by two seconds from the target duration. As with the previous experiments, it was assumed that shorter Es should result in shorter reproductions, whereas longer Es should lead to longer reproductions. Moreover, the number of $E$ s was varied. It was expected that the assimilation effect on reproductions would be stronger, the more $E$ s preceded the target interval. Thus, the difference between the target duration and the reproduction of the target duration should increase with increasing number of $E \mathrm{~s}$. In terms of analysis of variance, an interaction between number of $E \mathrm{~s}$ and duration of $E \mathrm{~s}$ was expected to occur. The values of the independent variable "duration of $E \mathrm{~s}$ " were 13 seconds and 17 seconds, the values of the independent variable "number of Es" were 2 and 20. Thus, four experimental conditions were realized.

\subsection{Method}

\subsubsection{Participants}

Sixteen men and 24 women with a mean age of 26.4 years $(S D=5.4)$ participated in Experiment 3. The participants were randomly and in equal number $(n=10)$ assigned to the four conditions.

\subsubsection{Materials and Procedure}

The stimuli and the procedure were identical to those of the previous experiments. However, changes were made according to the duration and the number of presentations of the expectancy intervals. The duration of the expectancy intervals was either $13 \mathrm{~s}$ or $17 \mathrm{~s}$. The number of presentations of the expectancy interval was either two or twenty. Thus, the experiment had a $2 \times 2$ factorial structure, with duration and number of expectancy intervals as independent variables.

\subsection{Results and Discussion}

Figure 3 displays the results of Experiment 3. The figure shows the mean reproduction times, plotted against the different durations and the different numbers of $E$ s.

Apparently, the duration of $E$ affected temporal reproductions, with shorter $E$ s yielding shorter reproductions than with longer $E$ s. Moreover, the number of preceding Es appeared to affect temporal reproductions as well. When only two $E$ s were presented, differences in mean reproductions between short and long expectancy intervals were smaller than when 20 Es were presented.

Analysis of variance, with duration and number of $E \mathrm{~s}$ as between-subject factors, and reproduction time as dependent variable, produced a significant main effect of duration of $E$, $F(1,36)=6.03, p=.019, \eta^{2}=.144$. Neither the main effect of number of presentations of $E$, nor the interaction effect, was significant, all $p \mathrm{~s}>.44$.

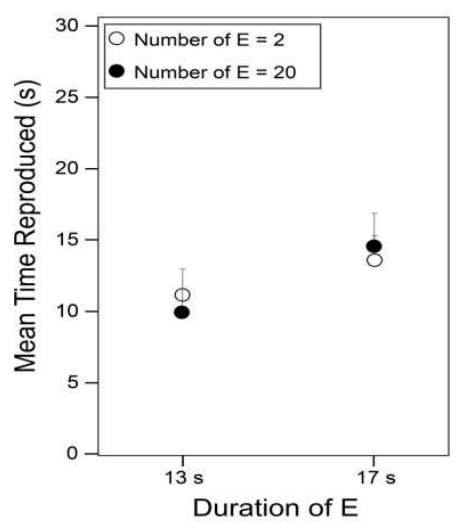

Figure 3. Mean reproduction times obtained from Experiment 3, plotted against the different $E$ durations (on the $x$-axis), and the different numbers of Es (indicated by open versus filled circles). Error bars mean standard errors.

Whereas the hypothesis of the occurrence of an assimilation effect could be confirmed, the hypothesis of different strengths of the assimilation effect due to different numbers of the presentation of the expectancy interval had to be rejected. Although visual inspection of Figure 3 suggests slightly more pronounced assimilation in case of a large quantity of repetitions of $E$ prior to the target interval, this difference did not reach statistical significance. Since only a small effect due to the number of presentations of $E$ was expected, the comparatively small number of individuals participating in this experiment might be responsible for not detecting the effect, if it had been present.

\section{Experiment 4}

In Experiment 4 a larger difference (10 seconds) between the duration of $E$ and the target duration was used. Again, it was expected that reproductions of the target duration should be biased by an assimilation effect. That is, reproductions should vary depending on the duration of the $E \mathrm{~s}$, with longer $E$ s resulting in longer reproductions, and vice versa. Moreover, compared to Experiment 3, this assimilation effect should be more pronounced when more $E$ s were induced prior to the target duration. As in Experiment 3, four conditions were realized, which differed regarding the duration of the $E$ s ( 5 seconds versus 25 seconds), and the number of $E$ s (2 versus 20).

\subsection{Method}

\subsubsection{Participants}

A total of 40 volunteers (twelve males and 28 females) participated, with a mean age of 24.3 years $(S D=4.8)$. The participants were randomly and in equal number $(n=10)$ assigned to the four conditions of the experiment.

\subsubsection{Materials and Procedure}

The stimuli and material used were the same as in Expe- 
riment 3, except for the durations of the $E$ s. The difference between the $E \mathrm{~s}$ and the target duration was raised to 10 seconds, which resulted in a short duration ( 5 seconds) and a long duration (25 seconds) of $E$.

The procedure of Experiment 4 was identical to that of the previous experiments.

\subsection{Results and Discussion}

The results of Experiment 4 are depicted in Figure 4. Obviously, there was a definite effect of $E$ on the reproductions of the target duration. Longer $E$ s yielded longer reproductions, and vice versa. Moreover, there was an interaction between numbers of $E \mathrm{~s}$ and duration of $E \mathrm{~s}$ : The assimilation effect, that is, the participants' tendency to reproduce $E$ rather than the target duration, was more pronounced with 20 Es than with 2 Es.

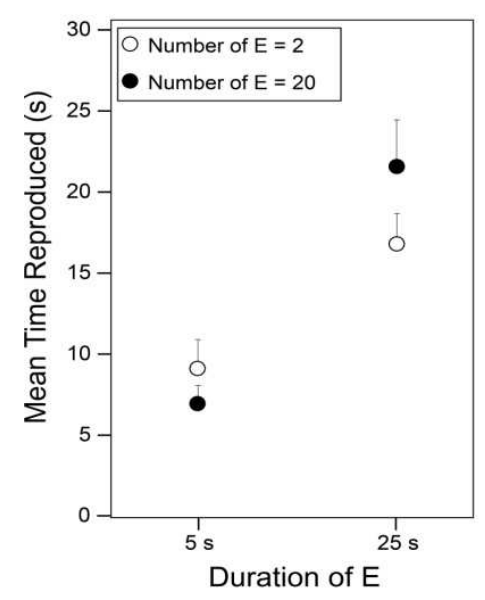

Figure 4. Mean reproduction times obtained from Experiment 4, plotted against the different $\mathrm{E}$ durations (on the $x$-axis), and the different numbers of Es (indicated by open versus filled circles). Error bars mean standard errors.

These apparent results were confirmed by analysis of variance, with duration and number of $E \mathrm{~s}$ as between-subject factors, and reproduction time as dependent variable. ANOVA yielded a significant main effect of duration of $E \mathrm{~s}$, $F(1,36)=45.36, p<.001, \eta^{2}=.558$, and a significant interaction, $F(1,36)=4.39, p=.043, \eta^{2}=.109$. The main effect of numbers of $E$ s was not significant, $F(1,36)=0.62$, $p=.437, \eta^{2}=.017$.

In line with the hypotheses stated, Experiment 4 revealed an assimilation effect on reproductions. Moreover, this assimilation effect was even more pronounced with a large number of $E$ s than with only two $E$ s. The latter effect might be due to a strengthening of the memory trace of the standard that has been used for comparison with the reproduced duration.

\section{Conclusions}

With this study, it was examined how temporal expectations modify retrospective reproductions of a target interval. Four experiments were conducted wherein the target interval was preceded by a sequence of expectancy intervals. The number of the expectancy intervals, as well as their duration was varied between participants. It was assumed that the preceding intervals should establish within an individual a mental representation of their duration which should in turn affect the reproduction of the target interval. The target interval was the last interval presented in the sequence. After the sequence of presented intervals had stopped, the participants were asked to reproduce the target interval. The reproduction task was retrospective, that is, the participants did not know in advance that they finally had to judge a time interval.

Results of the study indeed suggest that during the experiments the participants established a mental representation of the expectancy interval. According to adaptation-level theory, the standard that was finally used as a comparison within the reproduction task is supposed to be the average of all presented durations (including the target duration) that were experienced in the course of the experiment. The findings obtained further suggest that the representation of the expectancy intervals was strengthened with more expectancy intervals preceding the target interval. Thus, the more expectancy intervals were presented, the less were the reproductions affected by the target duration itself. Again, this result is in line with the assumption made by adaptation-level theory that the adaptation level (which might serve as a standard for temporal judgments) is the average of all intervals experienced within the experimental session.

However, no assimilation effect occurred when differences between preceding intervals and the target duration were rather small. This result might be due to a comparatively small sample size, related to an expected effect being also rather small.

In the following, an attempt is made to evaluate how well the empirical results of this study fit the outcomes predicted by adaptation-level theory. Adaptation-level theory suggests both the arithmetic and the geometric mean as a potential rule of aggregation of presented (and hence, perceived) durations towards an internal standard which is used for duration judgments. In Figure 5, the arithmetic and the geometric means of all durations presented (i. e., the adaptation levels) as well as the mean reproduction times are plotted against the different conditions realized in the four experiments. Note that the order of conditions, displayed on the x-axis, depends on the corresponding amount of the adaptation level, which is ascending.

Figure 5 shows that there is no remarkable difference between the quantification of the adaptation level as either the arithmetic or the geometric mean of the durations presented. Moreover, according to adaptation-level theory, mean reproductions should be based on the adaptation level that has been established within an experimental condition. This was apparently true for all conditions realized, as is indicated by the increase of mean reproductions that corresponds with an increase of the amount of adaptation level. However, mean reproductions were almost always shorter 
than the adaptation level, except for conditions yielding the shortest amounts of adaptation level. This result resembles many data gathered from temporal reproduction (and other time-estimation tasks) where shorter intervals were "overestimated" and longer intervals were "underestimated", a pattern of results that is known as "Vierordt's law" (e. g., [28]). There are different approaches reported in the literature that offer explanations for the different judgments of short and long durations. For instance, [29] and [30] describe the relationship between reproduced duration and standard duration as a power function with an exponent below one, which results in more or less accurate reproductions of short standards and shortened reproductions of long standards. Others assume limitations of short-term memory as the major source of this judgment bias (e. g., [31, 32]). This assumption is based on findings where intervals shorter or up to three seconds were reproduced correctly or slightly longer, whereas reproductions of intervals longer than approximately three seconds were on average shortened. Wearden [28] proposed that Vierordt-type effects might be due to both the duration of the motor response that is needed for making the judgment in temporal reproduction, and a decision threshold that defines at what difference between the standard and the presented duration an individual will start her motor response in order to terminate the duration presentation. In contrast to the short-term memory approach, Wearden's model does not imply a certain duration (the "indifference" duration) at which judgments are accurate and beyond which they become more and more inaccurate.

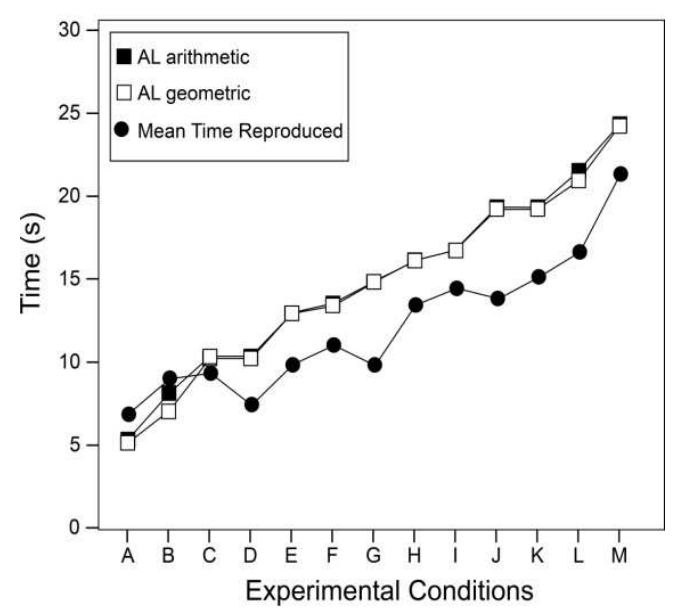

Figure 5. The adaptation levels, estimated by the arithmetic and the geometric means of all durations presented as well as the mean reproduction times, plotted against the different conditions realized in the four experiments. A: Exp. 4, $E=5 s, n=20 ; B: \operatorname{Exp} .4, E=5 s, n=2 ; C: \operatorname{Exp} .1, E=10 s$; D: $\operatorname{Exp} .2, E=10 s ; E: \operatorname{Exp} .3, E=13 s, n=20 ; F: \operatorname{Exp} .3, E=13 s, n=2 ; G$ : Exp. 2, $E=15 s ; H: \operatorname{Exp} .1, E=20 s ; I: \operatorname{Exp} .3, E=17 s, n=20 ; J: \operatorname{Exp} .3, E$ $=17 s, n=2 ; K: \operatorname{Exp} .2, E=20 s ; L: \operatorname{Exp} .4 ; E=25 s, n=2 ; M: \operatorname{Exp} .4, E=$ $25 s, n=20$.

Note: Exp. = Experiment; $E=$ Duration of expectancy intervals; $n=$ number of presentations of expectancy intervals.

The present results do rather not favour the short-term memory approach since even in conditions with the shortest "internal standards" the value of the adaptation level was above five seconds, which is far beyond what is usually assumed for the capacity of short-term memory. However, the data do not allow for establishing a detailed model of temporal reproductions, and this is surely a challenge for further studies.

Results of this study, therefore, call for further investigations. One the one hand, it would be interesting to examine why small differences between preceding intervals and the target interval did not elicit a clear assimilation effect. A straightforward way in attempting to get an answer to this question is simply to raise the sample size. Another possibility is offered by increasing the number of preceding intervals, as results of Experiment 3 suggested that with a large number of preceding intervals, at least a slight, yet not significant assimilation effect was observed. On the other hand, it is not clear if and how the duration of the periods of activity (in contrast to the expectancy intervals) contributed to temporal judgments. As the duration of these periods was held constant, a differential effect on duration judgments was unlikely to occur. Nonetheless, its duration may have played a role in establishing the internal standard for duration judgments. Therefore, in future studies, variation of the duration of the period of activity should be employed.

\section{Acknowledgements}

The author thanks Manuela Zirngibl for helping with data acquisition.

\section{References}

[1] J. T. Coull, and A. C. Nobre, "Dissociating explicit timing from temporal expectation with fMRI," Current Opinion in Neurobiology, vol. 18, pp. 137-144, 2008.

[2] L. G. Allan, "The perception of time," Perception \& Psychophysics, vol. 26, pp. 340-354, 1979.

[3] A. Hellstrom, "The time-order error and its relatives: Mirrors of cognitive processes in comparing," Psychological Bulletin, vol. 97 , pp. 35-61, 1985.

[4] K. Vierordt, Der Zeitsinn nach Versuchen [The time-sense according to experiments]. Tubingen, Germany: H. Laupp, 1868.

[5] H. Woodrow, "Time perception", in Handbook of experimental psychology, H. S. Langfeld (Ed.), New York: Wiley, 1951, pp. 1224-1236.

[6] R. Barnes, and M. R. Jones, "Expectancy, attention, and time," Cognitive Psychology, vol. 41, pp. 254-311, 2000.

[7] M. S. Berens, and R. E. Pastore, "Contextual relative temporal duration judgment: An investigation of sequence interruptions," Perception \& Psychophysics, vol. 67, pp. 102-119, 2005.

[8] K. S. Ahmadi, "Effects of social influences and waiting on time judgment," Perceptual and Motor Skills, vol. 59, pp. 771-776, 1984 
[9] M. K. Hui, and D. K. Tse, "What to tell consumers in waits of different lengths: An integrative model of service evaluation," Journal of Marketing, vol. 60, pp. 81-90, 1996.

[10] F. Klapproth, "Waiting as a temporal constraint," in Limits and constraints - The study of time XIII, J. A. Parker, P. Harris, and C. Steineck (Eds.), Leiden, The Netherlands, Brill, 2010, pp. 179-198.

[11] H. Helson, "Fundamental problems in color vision. I. The principle governing changes in hue, saturation, and lightness of non-selective samples in chromatic illumination," Journal of Experimental Psychology, vol. 23, pp. 439-476, 1938.

[12] H. Helson, "Adaptation-level as a basis for a quantitative theory of frames of reference," Psychological Review, vol. 55, pp. 297-313, 1948.

[13] H. Helson, Adaptation-level theory, New York: Harper \& Row, 1964.

[14] J. Capehart, V. J. Tempone, and J. A. Hébert, “A theory of stimulus equivalence," Psychological Review, vol. 76, pp. 405-418, 1969.

[15] D. R. Thomas, C. G. Jones, "Stimulus generalization as a function of the frame of reference," Journal of Experimental Psychology, vol. 64, pp. 77-80, 1962.

[16] D. R. Thomas, K. Mood, S. Morrison, and E. Wiertelak, "Peak shift revisited: A test of alternative interpretations," Journal of Experimental Psychology: Animal Behavior Processes, vol. 17, pp. 130-140, 1991.

[17] A. Parducci, "Sequential effects in judgment," Psychological Bulletin, vol. 61, pp. 163-167, 1964.

[18] D. R. Thomas, "A model for adaptation-level effects on stimulus generalization," Psychological Review, vol. 100, pp. 658-673, 1993.

[19] L. A. Bizo, and C. V. McMahon, "Temporal generalization and peak shift in humans," Learning \& Behavior, vol. 35, pp. 123-130, 2007.

[20] F. Klapproth, and M. Müller, "Temporal generalization under time pressure in humans," Quarterly Journal of Experimental Psychology, vol. 61, pp. 588-600, 2008.

[21] F. Klapproth, and J. H. Wearden, "Why do temporal generalization gradients change when people make decisions as quickly as possible?" Quarterly Journal of Experimental Psychology, vol. 64, pp. 1646-1664, 2011.

[22] J. Gibbon, R. M. Church, and W. H. Meck, "Scalar timing in memory," in Timing and time perception. Annals of the New York Academy of Sciences, vol. 423, J. Gibbon and L. Allan, Eds., 1984, pp. 52-77.

[23] A. Hellstrom, "Time errors are perceptual: An experimental investigation of duration and a quantitative successive-comparison model," Psychological Research, vol. 39, pp. 345-388, 1977.

[24] A. Hellstrom, "The time-order error and its relatives: Mirrors of cognitive processes in comparing," Psychological Bulletin, vol. 97, pp. 35-61, 1984.

[25] W. C. Michels, and H. Helson, H. "A quantitative theory of time-order effects," American Journal of Psychology, vol. 67, pp. 327-334, 1954 .

[26] S. W. Keele, R. Nicoletti, R. I. Ivry, R. I., and R. A. Pokorny, "Mechanisms of perceptual timing: Beat-based or interval-based judgments?" Psychological Research, vol. 50, pp. 251-256, 1989.

[27] R. Brickenkamp, D2-Aufmerksamkeits-Belastungs-Test [D2 attentional load test], Göttingen, Germany: Hogrefe, 2002.

[28] J. H. Wearden, "Applying the scalar timing model to human time psychology: Progress and challenges," in Time and mind II: Information processing perspectives, H. Helfrich (Ed.), Göttingen, Germany: Hogrefe \& Huber Publishers, 2003, pp. 21-39.

[29] H. Eisler, "Experiments on subjective duration 1868-1975: A collection of power function exponents," Psychological Bulletin, vol. 83, pp. 1154-1171, 1976.

[30] H. Eisler, and A. D. Eisler, "Subjective time scaling: Influence of age, gender, and type A and type B behaviour," Chronobiologia, vol. 21, pp. 185-200, 1994.

[31] E. Szelag, J. Kowalska, K. Rymarczyk, and E. Pöppel, “Duration processing in children as determined by time reproduction: Implications for a few seconds temporal window," Acta Psychologica, vol. 110, pp. 1-19, 2002.

[32] P. Ulbrich, J. Churan, M. Fink, and M. Wittmann, "Temporal reproduction: Further evidence for two processes," Acta Psychologica, vol. 125, pp. 51-65, 2007. 